'Departamento de Salud del Adulto y Senescente, Escuela de Enfermería, Pontificia Universidad Católica de Chile. Santiago, Chile.

${ }^{2}$ Departamento de Salud de la Mujer, Escuela de Enfermería, Pontificia Universidad Católica de Chile. Santiago, Chile.

${ }^{3}$ Centro Núcleo Milenio Autoridad y Asimetrías de Poder / Millennium Nucleus Center Authority and Power Asymmetries. Agencia Nacional de Investigación y Desarrollo. Chile.

${ }^{4}$ Dalla Lana School of Public Health, University of Toronto. Toronto, ON, Canadá. aEnfermera-matrona, Magíster en Psicología de la Salud, Pontificia Universidad Católica de Chile. Santiago, Chile.

${ }^{b}$ Enfermera, PhD (Medicine), Cardiff University.

'Psicóloga, doctoranda en Salud Pública, University of Toronto, Canadá.

dEnfermera- matrona, Magíster en Humanización en Salud, PhD Cuidado en Salud.

Financiamiento Agencia: Nacional de Investigación y Desarrollo ANID: Fondo Nacional de Investigación en Salud (FONIS), Proyecto FONIS SA1810002). FONIS no

influyó en el diseño del estudio, en la recolección, análisis o interpretación de los datos ni en la preparación, revisión o aprobación del manuscrito.

Las autoras declaran no tener ningún conflicto de interés.

Recibido el 13 de octubre de 2020, aceptado el 29 de diciembre de 2020

Correspondencia: Paulina Bravo

Av. Vicuña Mackena 4860, Macul. Santiago Chile.

pbbravo@uc.cl

\section{Consideraciones para comunicar riesgos y beneficios de la mamografía a mujeres desde la perspectiva de los expertos}

\author{
ANGELINA DOIS ${ }^{1, \mathrm{a}}$, PAULINA BRAVO ${ }^{2,3, \mathrm{~b}}$, \\ LORETO FERNÁNDEZ-GONZÁLEZ ${ }^{4, \mathrm{c}}$, CLAUDIA URIBE $^{2, \mathrm{~d}}$
}

\section{Experts' views on the communication of risks and benefits of mammography to detect breast cancer}

Background: Breast cancer ( $B C)$ has a high mortality rate in developing countries due to a scarcity of early detection. Risk communication is critical to support women who face the decision to undertake $B C$ screening. Thus, they can balance their perceived and real risk, and make informed choices. Aim: To describe experts' views on how the provision of information related to $B C$ screening should be made. Material and Methods: A qualitative study with focus groups with national experts was conducted. Open coding was performed. Results: Four categories on the way information about $B C$ screening should be provided emerged: to communicate about the need of the exam; the pros and cons of the test; fear as a barrier for understanding; and involving women in the decision-making process. Conclusions: These findings emphasize the need to include risk communication strategies in the patient-provider relationship and encourage and respect women's autonomy when facing the $B C$ screening decision.

(Rev Med Chile 2021; 149: 196-202)

Key words: Breast Neoplasms; Decision Making, Shared; Health Communication; Mammography.

\section{Jures,} a Organización Mundial de la Salud (OMS) ha establecido que el cáncer de mama es el tipo de neoplasia que más afecta a las mujeres, considerándolo un problema relevante de salud pública. Existe un significativo aumento de este cáncer en países en vías de desarrollo, donde la detección temprana es escasa y los hallazgos tardíos de la enfermedad acumulan importantes tasas de mortalidad ${ }^{1}$. En Chile, el cáncer de mama se ha convertido en la primera causa de muerte por tumores malignos en mujeres, acumulando una tasa de 12,9 por 100.000 habitantes ${ }^{2}$.
En respuesta a este panorama epidemiológico, los esfuerzos sanitarios se han centrado en la pesquisa temprana garantizada a través de pruebas de tamizaje. En esta línea, la mamografía se incluyó en las Garantías Explícitas en Salud (GES) como prestación en el nivel primario de atención, para mujeres entre 50 y 59 años ${ }^{3}$.

Si bien la mamografía es reconocida internacionalmente como el procedimiento de tamizaje de elección para el diagnóstico oportuno del cáncer de mama, en Chile, entre 20\% y 30\% de mujeres entre 50 y 59 años, no se realizan este exa- 
men $^{1,4}$. Considerando la relevancia del diagnóstico precoz de esta enfermedad para reducir el riesgo de muerte, el porcentaje de cobertura es insuficiente sobre todo al considerar grupos menos aventajados que pudieran acumular mayores factores de riesgo para este cáncer ${ }^{1,4-5}$.

En este escenario de baja cobertura es fundamental enfrentar los impedimentos para la realización del examen, detallados por las mismas mujeres. De acuerdo con reportes nacionales, las razones argumentadas se refieren al desconocimiento del examen, concentrando cerca de $40 \%$ de ellas en explicaciones como "no sabe que tiene que hacérselo", "no cree que lo necesita" y "no conoce el examen" 4 . Es por tanto fundamental, al definir estrategias de tamizaje y detección del cáncer de mama, que la mujer comprenda su propio riesgo de padecer o no la enfermedad y que los profesionales tengan la capacidad para transmitir con claridad y precisión la estimación del riesgo de cáncer de mama. Solo de esta manera la usuaria podrá tomar una decisión informada, que responda a su riesgo real de la enfermedad ${ }^{6}$.

La comunicación de riesgo implica proporcionar al paciente una síntesis de la evidencia acerca de las contingencias y beneficios asociados a un examen o tratamiento ${ }^{7}$, con el objetivo estratégico de disminuir la preocupación de las personas sobre daños potenciales relacionados a estos o a su condición de salud ${ }^{8}$. Con ello, se espera que las personas logren balancear el riesgo percibido con el riesgo real, de modo que sus conductas de salud sean razonables, certeras e informadas ${ }^{9}$.

En Chile, este tema no ha sido suficientemente explorado, por lo que el objetivo de este artículo es describir la opinión de profesionales expertos sobre qué consideraciones deben tomarse al entregar información a las mujeres que están enfrentando la decisión de realizarse o no una mamografía.

Los resultados que se presentan forman parte de la etapa de diseño del proyecto Herramienta para la toma de decisión informada de mamografía para usuarias del sistema público de atención de salud, financiado por el Fondo Nacional de Investigación en Salud (FONIS SA18I0002 de la Agencia Nacional de Investigación en Salud, Chile).

\section{Material y Método}

Estudio de diseño cualitativo, basado en la aproximación constructivista de la Grounded
Theory que se fundamenta en el supuesto que las personas interactúan basadas en la interpretación y significado que hacen de las experiencias y símbolos en sus vidas, asumiendo una postura reflexiva sobre los modos de saber y representarlo ${ }^{10}$.

Se realizaron grupos focales audiograbados con profesionales expertos en cáncer de mama de Santiago de Chile, para determinar los aspectos que ellos relevan como importantes para comunicar el riesgo asociado al tamizaje de cáncer de mama. El trabajo se concluyó cuando se alcanzó la saturación de los temas.

Para guiar la discusión se elaboró un guion temático con preguntas directrices relacionadas con cuál es la información esencial que necesitan las mujeres para tomar la decisión de realizarse o no el examen, cómo debería entregarse esa información y quién toma la decisión final.

Para la elección del grupo de expertos, se consideraron los criterios de la European Aid Cooperation Office: a) experiencia profesional (reconocida y respetada por sus pares); b) la independencia del evaluador y c) la capacidad de trabajo en equipo ${ }^{11}$. El marco muestral inicial estuvo formado por enfermeras, matronas, psicólogos, trabajadores sociales, médicos, gestores de salud de reconocida experiencia profesional que cumplieran los criterios de inclusión: profesionales de la salud involucrados en la indicación o realización de la mamografía o en el cuidado de mujeres en tratamiento del cáncer de mama al menos los últimos 5 años, y académicos que hubieran desarrollado líneas de trabajo e investigación vinculadas a cáncer de mama. La selección final del grupo participantes se hizo con muestreo por conveniencia y en cadena. Los grupos focales fueron transcritos y analizados en su totalidad. Para el análisis cualitativo se utilizó codificación abierta para identificar los conceptos emergentes, sus propiedades y dimensiones. Los pasos del proceso de análisis fueron: a) Lectura amplia y exhaustiva de las narrativas transcritas de cada grupo focal, para obtener un sentido del todo; b) Segunda lectura para identificar los temas o categorías relacionados con el fenómeno, fundamentadas en frases textuales; c) Organización de las categorías en torno a unidades de significado más amplias, para dar cuenta de la esencia del fenómeno en estudio ${ }^{12,13}$. El rigor metodológico se cauteló aplicando los criterios de Guba ${ }^{14}$.

El resguardo de la confidencialidad de la infor- 
mación, autonomía y dignidad de los participantes fue aprobado por el Comité Ético-Científico de la Facultad de Medicina de la Pontificia Universidad Católica de Chile (código 180313007).

\section{Resultados}

Se invitó a 21 profesionales que cumplían los criterios de inclusión. De ellos, 16 aceptaron participar. Las razones para no hacerlo se centraron mayormente en incompatibilidad horaria, con solo una profesional (tecnóloga médica), que explicitó sentir incomodidad con el tema del estudio. El grupo de expertos quedó formado por 12 profesionales (Tabla 1), que trabajaban en instituciones públicas y privadas (hospitalarias y ambulatorias) y representantes de una ONG.

A partir de la opinión de los expertos se describen cuatro categorías que dan cuenta de la

Tabla 1. Distribución participantes

\begin{tabular}{|lc|}
\hline Profesión & $\begin{array}{c}\text { Cantidad de } \\
\text { participantes }\end{array}$ \\
\hline Médico radiólogo & 2 \\
\hline Médico oncólogo & 1 \\
\hline Biólogo(a) & 1 \\
\hline Enfermero(a) & 3 \\
\hline Matrón (a) & 3 \\
\hline Psicooncólogo(a) & 1 \\
\hline Trabajador(a) social & 1 \\
\hline Total & 12 \\
\hline
\end{tabular}

información que debe entregarse a las mujeres que están enfrentando la decisión de realizarse o no una mamografía para la detección precoz de cáncer de mama:

\section{Categoría 1: Comunicar y dialogar sobre la nece- sidad del examen}

Los expertos coincidieron en dos aspectos a considerar en la comunicación profesional-usuario al momento de informar sobre la necesidad del examen: a) veracidad de la información, b) comunicación bidireccional horizontal entre las partes. Al respecto, recalcaron la importancia de la honestidad del contenido del mensaje (razones por las que se sugiere el examen a la mujer); así como el proporcionar un espacio para conversar sobre la necesidad de realizarse o no el examen para que la mujer pueda tomar una decisión informada (Tabla 2).

\section{Categoría 2: Presentación clara de las ventajas y desventajas del examen}

Los expertos coincidieron en que la principal ventaja de la mamografía es la posibilidad de hacer un diagnóstico precoz de cáncer de mama, incluso antes que sea clínicamente palpable, lo que se asocia a una mejor respuesta al tratamiento y al aumento de sobrevida de la mujer. Los participantes señalaron que, pese a lo controversial que puede ser el término, es importante explicar también la posibilidad de sobrediagnóstico que puede generarse con la mamografía. Este se entiende como la identificación de problemas de salud que no habrían causado daño a la persona. Sus principales causas se refieren a la sobredetección (identificación de anormalidades que no habrían

Tabla 2. Aspectos a considerar en la comunicación profesional-usuario

\begin{abstract}
"... hay que comunicarlo, pero de la mejor manera posible.... ¿Cómo?" (G1/169)1
"... en el fondo, tienes que ser honesto, honesto, porque claramente, aunque sea cáncer chiquitito, ya te dicen cáncer y, ya se te removió el piso, suena, suena feo" (G1/39)

"... porque lo que nos está pasando hasta ahora, en los consultorios y en los centros de salud ... es que la indicación de mamografía se da sin indicarla realmente, como quien dice hacerse un examen que es de rutina - decía la señora-. Entonces esa manera de entregar la información no entrega nada, ni siquiera la necesidad ..., entonces hay que hacer una entrega de la indicación de manera más dirigida ..." (G1/233)

"...la paciente te está preguntando - por qué me tengo que hacer este examen- ...yo creo que hay que escucharla, como que te está dejando ver algo" (G2/372)
\end{abstract}

${ }^{1}$ Las letras corresponden al grupo focal y el número que acompaña a la letra corresponde al párrafo de la transcripción donde aparece la cita. 
causado daño) y la sobredefinición de enfermedad (cambiando los límites para los factores de riesgo). Esto debe ser claramente comunicado a las mujeres, al momento de evaluar su riesgo personal de cáncer de mama y las consecuencias que podría tener realizarse o no el examen (Tabla 3 ).

\section{Categoría 3: El miedo como barrera que dificulta la comprensión de la información}

De acuerdo con la opinión de los participantes, al comunicar información relacionada con la mamografía se debe considerar el miedo como un factor que interviene en la decisión informada de la mujer. Se reconocen dos fuentes de miedo: a) Miedo al dolor que puede provocar el procedimiento; y b) Miedo al resultado del examen, relacionado con el cáncer y la muerte. En general las características de la información que reciben las mujeres no es una comunicación para reducir el temor, sino todo lo contrario, lo que la aleja de la posibilidad de tomar la decisión de hacerse el examen (Tabla 4).

\section{Categoría 4: Involucrar a la mujer en la toma de decisión}

En esta categoría los participantes coincidieron en la necesidad de promover y respetar la autonomía de la usuaria, tanto en la decisión de hacerse o no una mamografía, como en la decisión de tratar un cáncer si se detecta. Sin embargo, esa autonomía debe sostenerse en el acceso a información, en cantidad y calidad suficiente, para que la usuaria tome una decisión informada alineada con sus preferencias (Tabla 5).

\section{Tabla 3. Ventajas/desventajas del examen}

"... Claro que la recuperación suele ser buena, dependiendo de cuándo se diagnostique, eso, esa frase es importante, para que la gente tome conciencia de hacerse el examen..." (G1/14)

"Entonces es súper importante recalcar, que en el fondo es que el diagnóstico oportuno, eso es lo que tendrían que poner". $(\mathrm{G} 1 / 19)$

"El diagnóstico precoz, favorece la recuperación y las posibilidades de tratamiento pueden ser mayores..." (G2/166)

"pero yo creo que hay que ponerlo [el sobrediagnóstico], está en todas partes esta cuestión, digamos del sobrediagnosticado, aunque a ustedes [al resto del grupo] no les guste la palabra (G1/163)

\section{Tabla 4. Barreras para la comunicación}

... Yo digo, yo, poniéndome en el lugar de alguien que no tiene idea de lo que es cáncer ni nada de eso, y solamente se deja llevar por lo que se escucha en la tele, cáncer muerte todo. Al leerlo [en el texto revisado], pareciera que, si a mí ...la mamografía me da positivo, me da cáncer, voy a pasar por todo, eso de verdad que no..." (G2/254)

"ehhh lo, lo primero les digo, cuando a usted le dijeron cáncer de mama, ¿qué es lo primero que se les viene a la cabeza? -que me iba a morir- y muchas pacientes rechazan hacerse la mamografía, porque tienen miedo que les va a salir positiva" $(\mathrm{G} 2 / 159)$

"¿sabes cuál es el temor? por lo menos de las usuarias que habitualmente uno atiende, es la máquina, ya porque dicen "-No, me van aplastar la pechuga [la mama]-", ... pero la máquina provoca un poquito de rechazo al examen, cuando uno les dice le voy a dar una, le voy a dar la orden de la mamografía, por lo menos la experiencia, ehh, profesional que tengo en estos años, que eso provoca un poco de rechazo" (G2/32)

\section{Tabla 5. Toma de decisiones}

"Pero es que es una decisión [realizarse la mamografía], que tiene que tomar ella [la paciente]... " (G2/376)

"entonces te creo que una vez la mamografía te detecta una lesión, que es in situ qué se yo, o sea, entre comillas, resorte de la paciente o tratarla o no tratarla, manejarla, esperar, que, que el cuerpo le ayude..." (G1/168)

"Claro, ahora el derecho de la mamografía es cada 3 años, podríamos agregar que, la mujer podría solicitar otra con mayor frecuencia según los factores de riesgo" (G2/103) 


\section{Discusión}

Este artículo describe la opinión de profesionales expertos sobre qué incluir y cómo entregar la información a las mujeres que están enfrentando la decisión de realizarse, o no, la mamografía de tamizaje de cáncer de mama. Esto se ha organizado en cuatro categorías principales: 1) Comunicar y dialogar sobre la necesidad del examen; 2) Presentación clara de las ventajas y desventajas del examen; 3) El miedo como barrera que dificulta la comprensión de la información; y 4) Involucrar a la mujer en la toma de decisión.

Durante el encuentro clínico se produce un flujo informativo, entre el profesional y la usuaria, que coloca a la comunicación como un eje central en la atención sanitaria. Esto supone habilidades y capacidades de ambas partes para transmitir de manera efectiva un mensaje relevante para la situación de salud de la persona ${ }^{16}$.

Pese a los permanentes avances en salud, aún no es posible asegurar de manera absoluta qué es mejor para las personas frente a cualquier situación de salud-enfermedad ${ }^{16}$. En este escenario, surgen innumerables situaciones clínicas en que las personas enfrentan decisiones difíciles ${ }^{17}$. No existe un tratamiento médico que sea completamente seguro o libre de efectos no deseados. Este grado de incertidumbre significa que la decisión correcta dependerá estrechamente de las necesidades, valores y resultados esperados por las personas, en otras palabras, se transforma en una decisión "sensible a las preferencias" de la persona ${ }^{18}$. En este contexto, parte importante de la atención de salud se vincula a la comunicación de la incertidumbre propia de toda actividad médica ${ }^{19}$. Esto es especialmente relevante en el contexto de la mamografía. Los resultados de este estudio enfatizan sobre la necesidad de entregar a la usuaria un mensaje claro, honesto y basado en la mejor evidencia disponible (incluyendo el sobrediagnóstico como un resultado no deseado $)^{20}$, respecto de sus condiciones particulares de riesgo de cáncer de mama, de las características de la mamografía y de las ventajas y desventajas de realizarse el examen. Asimismo, dan cuenta de la necesidad de respetar y fomentar la autonomía de las usuarias para tomar decisiones relativas a su salud, mientras se reconoce el miedo que el examen puede generarles. Nuestros resultados son consistentes con la evidencia que muestra al miedo y fatalismo como barreras para la realización del examen ${ }^{21,22}$. Por ello, y para maximizar las ventajas que podrían obtenerse de la mamografía, es necesario implementar estrategias que permitan a la mujer hacer un juicio personal de su riesgo ${ }^{23}$, lo que podría conseguirse a través de herramientas de apoyo para la toma de decisión en salud. Estas herramientas deben construirse y validarse en procesos de investigación rigurosos, que incluyan la medición de la toma de decisión informada de mamografía ${ }^{24}$.

Los resultados obtenidos se alinean con los requisitos definidos para la comunicación de riesgo, como, por ejemplo, que la información entregada a la usuaria considere todos los puntos de vista, ofrecer un trato respetuoso que dé respuesta a todas las partes implicadas, reconocer las necesidades específicas de cada persona que le permitan juzgar el nivel de confianza y la responsabilidad de quien comunica, entregar información basada en evidencia científica y hacer de la percepción del riesgo un proceso más participativo, democrático, transparente y accesible ${ }^{8}$. De este modo, a pesar de que en Chile no existe entrenamiento específico en comunicación de riesgo, los profesionales son capaces de identificar su importancia y asociar de forma intuitiva esta dimensión a las buenas prácticas de comunicación en salud ${ }^{25}$.

Dentro de las limitaciones del presente estudio, destacan que solo se reclutaron profesionales de Santiago de Chile, dejando fuera posibles voces de otras regiones del país. Además, no fue posible contar con la participación de tecnólogos(as) médicos(as), a pesar de los esfuerzos realizados, lo que puede abordarse en futuras pesquisas buscando dirigidamente la opinión de ese grupo de profesionales.

En particular, observamos que el tema del estudio fue la razón de rechazo a participar en al menos una de estas profesionales, lo que da cuenta que es un tema que concierne las creencias y valores de los profesionales respecto de lo que consideran beneficioso en torno a la mamografía. Esto podría constituirse en una barrera para la implementación de intervenciones que promuevan el involucramiento de las personas en las decisiones sanitarias, puesto que el acceso a información no asegura que puedan tener un rol activo, ya que para este se necesita que el profesional se desprenda de lo que cree es la mejor opción clínica 
y facilite que la persona identifique aquello que valora y prefiere de acuerdo con su contexto y particularidad $^{26}$.

\section{Conclusión}

El actual escenario tecnológico favorece la comunicación y la inmediatez en la circulación de la información, lo que hace que la población tenga un acceso rápido a la misma y que, además, demande respuestas razonadas y razonables ante situaciones que pudieran vincularse a un mayor riesgo de su salud. Ser conscientes de este cambio es una oportunidad para los equipos de salud, y por ello es relevante que los profesionales de salud comprendan los factores de riesgo, tanto los asociados al cáncer de mama, como los de su tamizaje. De este modo, se podrán enfrentar las dificultades al indicar de manera oportuna la mamografía y entregar información relevante para que la mujer pueda realizar una decisión informada basada en la mejor evidencia disponible.

Los resultados muestran que los profesionales que trabajan en el área consideran importante la comunicación y diálogo sobre la indicación de mamografía, presentando las ventajas y desventajas de esta, abordando el miedo que puede despertar el examen y respetando la autonomía de la mujer. Si bien, la sola entrega de información no es suficiente para promover una acción en salud, existe suficiente evidencia que apoya el hecho que, a mayor información, mayor es la comprensión del riesgo y, por lo tanto, mayor la autoeficacia para tomar decisiones respecto de la propia salud.

\section{Referencias}

1. World Health Organization [internet]. Ginebra; Breast cancer: prevention and control; 2017 Disponible en http://www.who.int/cancer/detection/breastcancer/en/] [Consultado el 13 de octubre de 2020].

2. Icaza G, Núñez L, Bugueño H. Descripción epidemiológica de la mortalidad por cáncer de mama en mujeres en Chile. Rev Med Chile 2017; 145 (1): 106-14. Disponible en: https://scielo.conicyt.cl/scielo.php?script=sci_arttext\&pid=S0034-98872017000100014\&lng=es. http:// dx.doi.org/10.4067/S0034-98872017000100014. [Consultado el 13 de octubre de 2020].

3. Ministerio de Salud de Chile M. [internet] Santiago,
Chile; Estrategia nacional de cáncer. Disponible en https://www.minsal.cl/wp-content/uploads/2016/10/Estrategia-Nacional-de-Cancer-version-consulta-publica. pdf. [Consultado el 13 de octubre de 2020].

4. Ministerio de Desarrollo Social [internet] Santiago, Chile; Encuesta de Caracterización Socioeconómica Nacional (CASEN 2015). Síntesis de resultados de salud. 2016. Disponible en http://www.desarrollosocialyfamilia.gob. cl/pdf/upload/IDS_INAL_FCM_3.pdf . [Consultado el 13 de octubre de 2020].

5. Cochrane Database Syst Rev. 2013 [internet]. Gartlehner G, Thaler K, Chapman A, Kaminski-Hartenthaler A, Berzaczy D, Van Noord MG, et al. T. Mammography in combination with breast ultrasonography versus mammography for breast cancer screening in women at average risk. Cochrane database of systematic reviews. Disponible en https://pubmed.ncbi.nlm.nih. gov/23633376/ [Consultado el 13 de octubre de 2020]

6. Ghosh K, Crawford BJ, Pruthi S, Williams CI, Neal L, Sandhu NP, et al. Frequency format diagram and probability chart for breast cancer risk communication: a prospective, randomized trial. BMC women's health 2008; 8: 18. Disponible en https://bmcwomenshealth. biomedcentral.com/articles/10.1186/1472-6874-8-18. [Consultado el 13 de octubre de 2020].

7. Ahmed H, Naik G, Willoughby H, Edwards AGK. Communicating risk. BMJ 2012; 344: e3996. Disponible en https://www.bmj.com/content/344/bmj.e3996. [Consultado el 13 de octubre de 2020].

8. Tormo MJ, Banegas JR. Mejorar la comunicación de riesgos en Salud Pública: sin tiempo para demoras. Rev Esp. Salud Pública 2001; 75 (1): 7-10. Disponible en http://scielo.isciii.es/scielo.php?script=sci_arttext\&pi$\mathrm{d}=$ S1135-57272001000100001 [Consultado el 13 de octubre de 2020].

9. Edwards AG, Naik G, Ahmed H, Elwyn GJ, Pickles T, Hood K, et al. Personalised risk communication for informed decision making about taking screening tests. Cochrane Database Syst Rev. 2016 [internet]. Disponible en: https://pubmed.ncbi.nlm.nih.gov/23450534/ [Consultado el 13 de octubre de 2020]

10. Charmaz K. Grounded theory in the $21^{\text {st }}$ century: Applications for social justice studies. En: Denzin N \& Lincoln Y, Editores Handbook of qualitative research Thousand Oaks, CA: Sage. 2005; 507-35.

11. García V, Aquino S, Guzmán A, Medina A. El uso del método Delphi como estrategia para la valoración de indicadores de calidad en programas educativos a distancia. Revista CAES 2012; 3 (1): 200-22. Disponible en https://dialnet.unirioja.es/servlet/articulo?codigo=3945837 [Consultado el 13 de octubre de 2020]. 
12. Strauss A, Corbin J. Basics of qualitative research. California, EE. UU: Sage; 1990.

13. Flick U. Introducción a la investigación cualitativa. Madrid, España: Morata; 2004.

14. Guba E. The paradigm dialog. Newbury Park, Estados Unidos: Sage; 1990

15. Brodersen J, Schwartz LM, Heneghan C, O'Sullivan JW, Aronson JK, Woloshin S. Overdiagnosis: what it is and what it isn't. BMJ Evidence-Based Medicine 2018; 23: $1-3$.

16. Bravo P, Contreras A, Perestelo-Pérez L, Pérez-Ramos J, Málaga G. En busca de una salud más participativa: compartiendo decisiones de salud. Rev Peru Med Exp Salud Publica 2013; 30 (4): 691-7. Disponible en http://www.scielo.org.pe/scielo.php?script=sci_arttext\&pid=S1726-46342013000400025\&lng=es\&nrm=iso [Consultado el 04 de abril de 2020].

17. Cochrane Database Syst Rev. 2017 [internet]. O'Connor AM, Bennett CL, Stacey D, Barry M, Col NF, Eden KB, et al. Decision aids for people facing health treatment or screening decisions Disponible en: https://www. cochrane.org/CD001431/COMMUN_decision-aidshelp-people-who-are-facing-health-treatment-or-screening-decisions [Consultado el 13 de octubre de 2020].

18. Coulter A, Collins A. Making shared decision-making a reality: no decision about me, without me. Londres, Reino Unido: The King's Fund; 2011.

19. Dolan JG, Qian F, Veazie PJ. How well do commonly used data presentation formats support comparative effectiveness evaluations? Med Decis Making 2012; 32 (6): 840-50. Disponible en doi: 10.1177/0272989X12445284 [Consultado el 04 de abril de 2020].

20. Zarazaga A, Franco-López A, Culebras JM. Benefits and damages of Screening. Overdiagnosis and anticipatory medicine. JONNPR 2020; 5 (4): 369-78. Disponible en http://scielo.isciii.es/scielo.php?script=sci_arttext\&pid=S2529-850X2020000400002\&lng=es. Epub
14-Sep-2020. http://dx.doi.org/10.19230/jonnpr.3299 [Consultado el 13 de octubre de 2020].

21. Peek ME, Sayad JV, Markwardt R. Fear, fatalism and breast cancer screening in low-income African-American women: the role of clinicians and the health care system. J Gen Intern Med 2008; 23 (11): 1847-53. Disponible en https://www.ncbi.nlm.nih.gov/pmc/articles/ PMC2585682/ [Consultado el 13 de octubre de 2020].

22. Kissal A, Vural B, Ersin F, Solmaz T. The effect of women's breast cancer fear and social support perceptions on the process of participating in screening. Glob Health Promot 2018; 25 (3): 52-9. Disponible en https://pubmed.ncbi.nlm.nih.gov/28195527/ [Consultado el 13 de octubre de 2020].

23. Pace LE, Keating NL. A Systematic Assessment of Benefits and Risks to Guide Breast Cancer Screening Decisions. JAMA 2014; 311 (13): 1327-35. Disponible en doi: 10.1001/jama.2014.1398. [Consultado el 13 de octubre de 2020].

24. Hersch J, McCaffery K. Using a decision aid may prompt some younger women (38-50 years) to rethink breast cancer screening plans. Evidence-Based Nursing 2018; 21:10-1. Disponible en http://dx.doi.org/10.1136/eb2017-102705 [Consultado el 13 de octubre de 2020].

25. Dois A, Bravo P, Soto G. Atributos y características de los principios orientadores del Modelo de Atención Integral de Salud Familiar y Comunitaria desde la perspectiva de expertos en APS. Rev Med Chile 2017; 145 (7): 743-51. Disponible en https://scielo.conicyt.cl/scielo.php?scrip$\mathrm{t}=$ sci_arttext\&pid=S0034-98872017000700879\&lng=es. http://dx.doi.org/10.4067/s0034-98872017000700879. [Consultado el 13 de octubre de 2020].

26. Joseph-Williams N, Elwyn G, Edwards A. Knowledge is not power for patients: a systematic review and thematic synthesis of patient-reported barriers and facilitators to shared decision making. Patient Educ Couns 2014; 94 (3): 291-309. Disponible en doi: 10.1016/j. pec.2013.10.031. [Consultado el 13 de octubre de 2020]. 